\title{
Physical properties of two compact high-velocity clouds possibly associated with the leading arm of the Magellanic system ${ }^{\star}$
}

\author{
N. Ben Bekhti, C. Brüns, J. Kerp, and T. Westmeier \\ Argelander-Institut für Astronomie ${ }^{\star \star}$, Universität Bonn, Auf dem Hügel 71, 53121 Bonn, Germany \\ e-mail: nbekhti@astro.uni-bonn.de
}

Received 17 February 2006 / Accepted 7 July 2006

ABSTRACT

\begin{abstract}
Aims. We observed two compact high-velocity clouds HVC 291+26+195 and HVC 297+09+253 to analyse their structure, dynamics, and physical parameters. In both cases there is evidence for an association with the Leading Arm of the Magellanic Clouds. The goal of our study is to learn more about the origin of the two CHVCs and to use them as probes for the structure and evolution of the Leading Arm.

Methods. We have used the Parkes 64-m radio telescope and the Australia Telescope Compact Array (ATCA) to study the two CHVCs in the $21-\mathrm{cm}$ line emission of neutral hydrogen. The observations with a single-dish and a synthesis telescope allow us to analyse both the diffuse, extended emission as well as the small-scale structure of the clouds. We present a method to estimate the distance of the two CHVCs.

Results. The investigation of the line profiles of HVC 297+09+253 reveals the presence of two line components in the spectra which can be identified with a cold and a warm gas phase. In addition, we find a distinct head-tail structure in combination with a radial velocity gradient along the tail, suggesting a ram-pressure interaction of this cloud with an ambient medium. HVC 291+26+195 has only a cold gas phase and no head-tail structure. The ATCA data show several cold, compact clumps in both clouds which, in the case of HVC $297+09+253$, are embedded in the warm, diffuse envelope. All these clumps have very narrow H lines with typical line widths between 2 and $4 \mathrm{~km} \mathrm{~s}^{-1} F W H M$, yielding an upper limit for the kinetic temperature of the gas of $T_{\max }=300 \mathrm{~K}$. We obtain distance estimates for both CHVCs of the order of 10 to $60 \mathrm{kpc}$, providing additional evidence for an association of the clouds with the Leading Arm. Assuming a distance of $50 \mathrm{kpc}$, we get Hi masses of $5.9 \times 10^{3} M_{\odot}$ and $4.0 \times 10^{4} M_{\odot}$ for HVC $291+26+195$ and HVC 297+09+253, respectively.
\end{abstract}

Key words. Galaxy: halo - ISM: clouds - ISM: structure - ISM: kinematics and dynamics - galaxies: Magellanic Clouds

\section{Introduction}

High-velocity clouds (HVC, Muller et al. 1963) are clouds of neutral atomic hydrogen, with radial velocities $\left(\left|v_{\mathrm{LSR}}\right| \gtrsim\right.$ $100 \mathrm{~km} \mathrm{~s}^{-1}$ ) inconsistent with a simple model of galactic rotation. They are believed to be extraplanar objects located in the halo (e.g. complex A, van Woerden et al. 1999) and circumgalactic environment of disk galaxies (e.g. the Magellanic Stream, Mathewson et al. 1974). Wakker (1991) introduced the so-called deviation velocity, $v_{\mathrm{dev}}$, that is the difference between the velocity of the clouds in the LSR frame and the extreme velocity value consistent with the Milky Way gas distributed along the line of sight. A cloud is defined as an HVC, if $\left|v_{\text {dev }}\right| \geq 50 \mathrm{~km} \mathrm{~s}^{-1}$.

HVCs can be detected all over the sky, but they are not homogeneously distributed (Wakker \& van Woerden 1997; Murphy et al. 1995; de Heij et al. 2002). On the one hand, there are coherent and extended objects like the complexes A, C and M and the Magellanic Stream. On the other hand, there are compact, isolated clouds with angular diameters of $\varphi \leq 2^{\circ} F W H M$,

^ The Australia Telescope Compact Array and the Parkes telescope are part of the Australia Telescope which is funded by the Commonwealth of Australia for operation as a National Facility managed by CSIRO.

$\star \star$ Founded by merging of the Institut für Astrophysik und Extraterrestrische Forschung, the Sternwarte, and the Radioastronomisches Institut der Universität Bonn. the so-called compact high-velocity clouds (CHVCs), which are kinematically and spatially separated from the gas distribution in their environment (Braun \& Burton 1999).

The most critical issue of $\mathrm{HVC}$ research is the determination of distances. It is very difficult to find suitable background sources at known distances against which the clouds would appear in absorption. Due to this fact the spatial distribution of HVCs is largely unknown, and important distance-dependent physical parameters, like mass $\left(M \sim d^{2}\right)$, radius $(R \sim d)$ and particle density $\left(n \sim d^{-1}\right)$, are poorly constrained. The distances of only three complexes have been determined so far via absorption line measurements. For complex $\mathrm{M}$ a distance of $1.5<z<4.4 \mathrm{kpc}$ was obtained by Danly et al. (1993), and a distance bracket for complex A of $4<z<10 \mathrm{kpc}$ was determined by van Woerden et al. (1999). Recently, Thom et al. (2006) derived a distance bracket for HVC complex WB of $7.7<d<8.8 \mathrm{kpc}$.

There are three main hypotheses for the origin of HVCs. The first is the formation from gas flowing out of the Galactic disk (galactic fountain model, Shapiro \& Field 1976; Bregman 1980), the second is the origin from the interaction of dwarf galaxies with the Milky Way (Gardiner \& Noguchi 1996; Bland-Hawthorn et al. 1998; Bregman 2004), and the third hypotheses is the primordial gas model, where HVCs represent intergalactic gas which was not yet accreted by one of the galaxies in the Local Group (Oort 1966; Blitz et al. 1999). 
Several compact high-velocity clouds (CHVCs) have been studied in detail (Braun \& Burton 2000; Burton et al. 2001; Westmeier et al. 2005). In many cases, two-component line profiles are observed which can be identified with a warm and a cold gas phase (Giovanelli et al. 1973; Greisen \& Cram 1976; Wolfire et al. 1995). Furthermore, some clouds show a head-tail structure or horse-shoe shape connected with gradients in column density and in line width across the symmetry axis. These observations suggest an interaction of the clouds with their ambient medium. Interferometric observations (Braun \& Burton 2000; de Heij et al. 2002) revealed that these clouds have a characteristic morphology. Compact cores are embedded in diffuse warm H I gas. The clumpy substructures have relatively high column densities $\left(\approx 10^{20} \mathrm{~cm}^{-2}\right)$ and very small line widths down to $2 \mathrm{~km} \mathrm{~s}^{-1} F W H M$. This indicates that the compact cores can be identified with a cool neutral medium.

Recently, a statistical analysis of the high-velocity sky was carried out by Kalberla \& Haud (2006) based on the Leiden/Argentine/Bonn (LAB) Galactic H I Survey (Kalberla et al. 2005). They searched all HVC complexes for indications of multi-phase structures, excluding CHVCs which are not sufficiently resolved by the LAB Survey. A multi-phase structure was found in $f_{\mathrm{m}}=23.8 \%$ of all sight lines investigated by Kalberla \& Haud (2006), corresponding to a contribution of the cold cores to the total H I column density of $f_{N}=20.8 \%$. Thus, a multi-phase structure is a common phenomenon in high-velocity clouds which means that many HVCs contain cold, compact cores embedded in a diffuse envelope of warm neutral gas. Two-component line profiles are also frequent in the Magellanic System. In the Leading Arm $f_{\mathrm{m}}=23.8 \%$ of sight lines with HVC emssion show a multi-phase structure, equivalent to a column density contribution of the cold gas of $f_{N}=13.5 \%$. The corresponding numbers for the Magellanic Stream are $f_{\mathrm{m}}=38.0 \%$ and $f_{N}=27.0 \%$ for the region with positive radial velocities but only $f_{\mathrm{m}}=7.5 \%$ and $f_{N}=11.0 \%$ for the negative-velocity region. The multi-component structure of the Leading Arm is confirmed by $\mathrm{H}$ I observations with the Parkes telescope and the ATCA by Brüns et al. (2005) and Brüns \& Westmeier (2004).

Wakker et al. (2002) studied a denser region in one of the filaments of the Leading Arm of the Magellanic System using the Parkes telescope and the ATCA. Their data reveal a very complex internal structure of the high-velocity gas. The filamentary structure introduces a high degree of complexity which does not allow to draw conclusions about the physical conditions in the Leading Arm and its environment.

We decided to study two compact clouds, HVC 291+26+195 and HVC $297+09+253$, which are isolated but located in the vicinity of the Leading Arm. Figure 1 shows a column density map of the Magellanic System (Brüns et al. 2005). The two circles mark the positions of the two CHVCs. Both clouds were observed with the Parkes telescope and the ATCA. The observations with both a single-dish telescope and an interferometer allow us to analyse the total H I mass and the extended structure as well as the small-scale structure within the clouds. These clouds are supposed to have a relatively simple structure, allowing us to uncover their physical state and that of the ambient medium. If the CHVCs were associated with the Leading Arm we would be able to roughly constrain their distance and origin, which are crucial but unknown for most HVCs/CHVCs. This would allow us to determine distance-dependent physical parameters such as mass and density and to study the interaction effects observed in connection with the Leading Arm. Furthermore, with the highresolution synthesis observations we are able to investigate a possible multi-component structure in both clouds which were

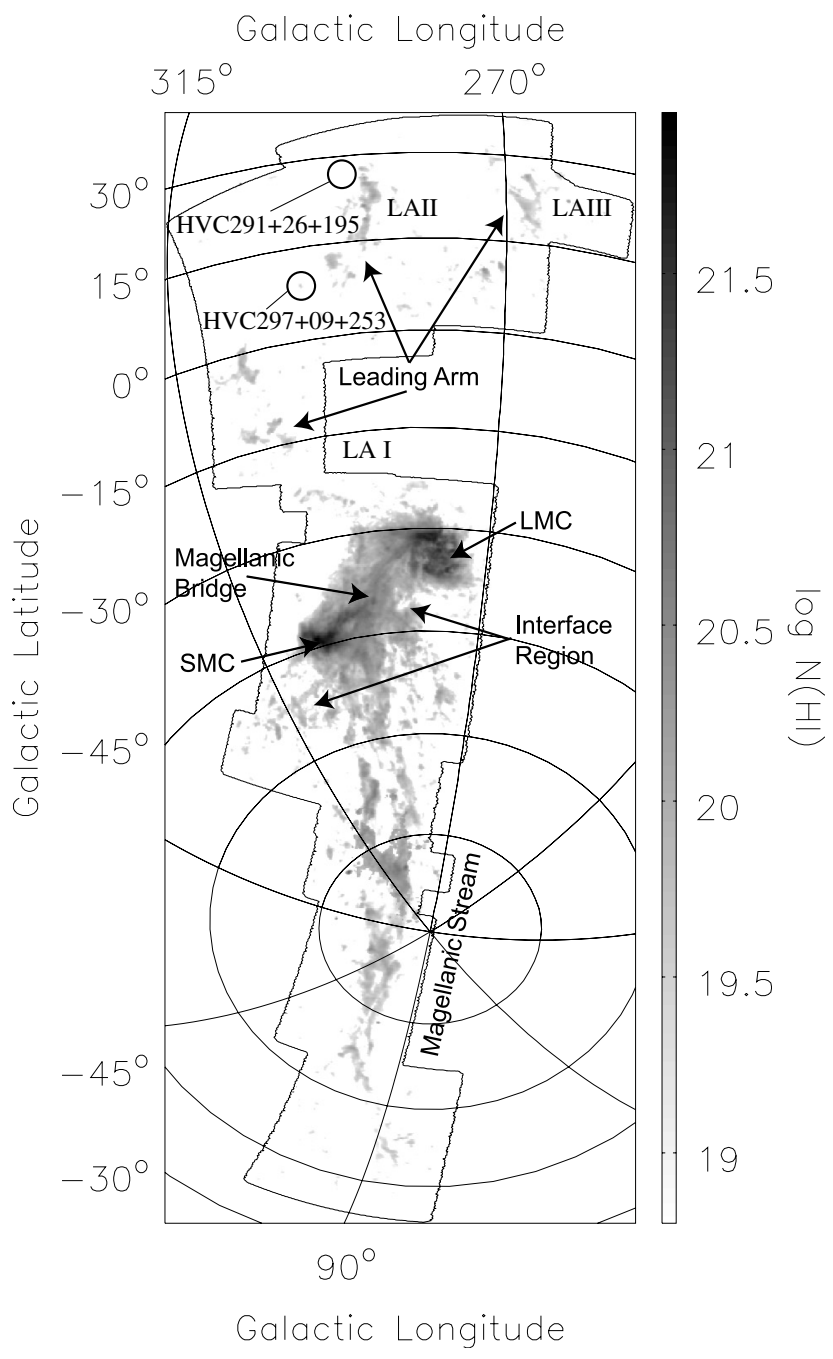

Fig. 1. H I column density distribution of the Magellanic System from Brüns et al. (2005). The column densities range from $N_{\mathrm{HI}}=6 \times$ $10^{18} \mathrm{~cm}^{-2}$ (light grey) to $N_{\mathrm{HI}}=9 \times 10^{21} \mathrm{~cm}^{-2}$ (black). The black circles mark the positions of HVC $297+09+253$ and HVC $291+26+195$.

not included in the sample of Kalberla \& Haud (2006) since the spatial resolution of the LAB Survey is not adequate to resolve them.

Our paper is organised as follows. In Sect. 2 we describe the data acquisition and data reduction. In Sect. 3 the results of the analysis of the Parkes and the ATCA data of both CHVCs are presented. In Sect. 4 we discuss the results regarding rampressure interaction, a distance estimate and a probable association with the Leading Arm of the Magellanic System. Section 5 summarises our results and point out the importance of followup observations that will improve our understanding of the nature of high-velocity clouds.

\section{Data acquisition and reduction}

\subsection{Parkes data}

The Parkes data were obtained as part of an H I survey of the Magellanic System (Brüns et al. 2005). The Parkes telescope has a diameter of $64 \mathrm{~m}$. At $21 \mathrm{~cm}$ wavelength the half-power beam width (HPBW) is 14 ! 1 . For our observation the velocity resolution is about $1 \mathrm{~km} \mathrm{~s}^{-1}$. The width of a spectral bin corresponds to $0.825 \mathrm{~km} \mathrm{~s}^{-1}$ using the Parkes 2048-channel autocorrelator. 
The H I data were observed in on-the-fly mode. The integration time per spectrum was $5 \mathrm{~s}$ and the beam moved $5^{\prime}$ on the sky during the integration (Brüns et al. 2005).

The single-dish data were analysed with the GILDAS software CLASS. In all spectra polynomial baselines up to 5th order were fitted. For this purpose windows were set individually around the line emission. The data within these windows were not considered for the fit. After the baseline fit Gaussian functions were fitted in the spectral lines. The criterion for line emission was set to $3 \sigma_{\mathrm{rms}}$ at a velocity resolution of $2 \mathrm{~km} \mathrm{~s}^{-1}$. The noise of the Parkes data is about $\sigma_{\text {rms }}=0.1 \mathrm{~K}$. For the further data analysis data cubes for both clouds were prepared.

\subsection{ATCA data}

The ATCA is an east-west interferometer with six antennas. Each antenna has a diameter of $22 \mathrm{~m}$. Of these six antennas only five were used for the following analysis. The ATCA data were observed using the 750D configuration. For this configuration the five antennas provide baselines between $30 \mathrm{~m}$ and $720 \mathrm{~m}$. The baselines associated with the sixth antenna correspond to very small angular scales, where no signal is detected. For our observations we chose a correlator with a bandwidth of $4 \mathrm{MHz}$ and a velocity resolution of about $0.8 \mathrm{~km} \mathrm{~s}^{-1}$. The width of a spectral channel is $0.8 \mathrm{~km} \mathrm{~s}^{-1}$ with a total of 1024 channels. The observing time for each cloud was $12.5 \mathrm{~h}$.

The ATCA data were analysed with the MIRIAD software. The source 1934-638 was used as primary calibrator for the flux calibration. The primary calibrator was observed for about $15 \mathrm{~min}$ each at the beginning and at the end of the observation. The secondary calibrator, 1215-457, was used for the gain and bandpass calibration, this source was observed once every hour for $5 \mathrm{~min}$. The pointing centre is $\alpha(\mathrm{J} 2000)=12^{\mathrm{h}} 12^{\mathrm{m}} 35^{\mathrm{s}}$, $\delta(\mathrm{J} 2000)=-53^{\circ} 42^{\prime} 30^{\prime \prime}$ for HVC $297+09+253$ and $\alpha(\mathrm{J} 2000)=$ $11^{\mathrm{h}} 58^{\mathrm{m}} 14^{\mathrm{s}}, \delta(\mathrm{J} 2000)=-35^{\circ} 34^{\prime} 36^{\prime \prime}$ for HVC $291+26+195$. We used a robustness parameter of 0.5 to produce a data cube. This robustness parameter is a compromise between a good resolution and a high signal-to-noise ratio $(S N R)$. The image size of one plane of the data cube is $300 \times 300$ pixels, the pixel size is $8^{\prime \prime}$. The HPBW of the elliptical beam for the corresponding parameters is about $58^{\prime \prime} \times 36^{\prime \prime}$ for HVC $297+09+253$ and $75^{\prime \prime} \times 38^{\prime \prime}$ for HVC $291+26+195$. The deconvolution was performed with the CLEAN algorithm (Högbom 1974). The final data cube has an rms of about $7.6 \mathrm{mJy}^{\text {beam }}{ }^{-1}$ towards the centre of the field which corresponds to $1.7 \mathrm{~K}$.

\section{Results}

\subsection{HVC $297+09+253$}

\subsubsection{Parkes data}

Figure 2a shows a column density map of the Parkes data of HVC 297+09+253. The CHVC shows an elongated head-tail structure with a major axis of about $1^{\circ}$ and a minor axis that is not resolved by the Parkes beam.

The average of all 59 spectra of the CHVC reveals a twocomponent line structure, where a narrow line component is superposed on a broad line component (Fig. 2b). The figure also shows the results of a two-component Gaussian fit to the average spectrum. The line profile is well represented by two Gaussians. The Gaussian fits provide line widths of $20.9 \pm 0.6 \mathrm{~km} \mathrm{~s}^{-1} F W H M$ for the broad component and $4.2 \pm 0.2 \mathrm{~km} \mathrm{~s}^{-1} F W H M$ for the narrow component. The so-called Doppler temperature assumes a pure Maxwellian velocity distribution and provides an upper temperature limit for the gas

$\frac{T_{\mathrm{D}}}{[\mathrm{K}]}=21.8\left(\frac{\Delta v_{F W H M}}{\left[\mathrm{~km} \mathrm{~s}^{-1}\right]}\right)^{2}$,

where $\Delta v_{F W H M}$ is the line width. The resulting upper temperature limits are $9500 \pm 550 \mathrm{~K}$ (broad component) and $380 \pm 40 \mathrm{~K}$ (narrow component). The kinetic temperatures of the two gas phases are expected to be lower, as e.g. turbulent motions also contribute to the observed line width.

For a more detailed analysis of the physical parameters of the two gas phases Gaussian fits were performed for the individual spectra of HVC 297+09+253. Two Gaussian components were fitted to those spectra that are not well represented by a single Gaussian. Figure 2c shows a histogram of the line widths resulting from the Gaussian decomposition. There are two separate distributions with maxima at $5 \mathrm{~km} \mathrm{~s}^{-1} F W H M$ for the narrow component and about $20 \mathrm{~km} \mathrm{~s}^{-1} F W H M$ for the broad line component. The histogram implies that a line width of about $10 \mathrm{~km} \mathrm{~s}^{-1}$ provides an adequate separation between the two gas components. This line width corresponds to a temperature of $2000 \mathrm{~K}$.

The distribution of the parameters $N_{\mathrm{HI}}, v_{\mathrm{LSR}}$ and $\Delta v_{F W H M}$ is analysed along the major axis of HVC $297+09+253$ (white line in Fig. 2a), and the results are shown in Figs. 2d-f. The diagrams demonstrate that the two gas phases are partly spatially separated. The maxima of the column density of the two line components are displaced by $\approx 15^{\prime}$. The narrow component is very compact with a $F W H M$ diameter of only $\approx 15^{\prime}$ which is similar to the Parkes HPBW of 14 .'1, indicating that the cold component is not resolved by the Parkes telescope. The column density of the warm component rises steeply close to the cold component and decreases slowly towards the southern end of HVC $297+09+253$. The spatial distribution of the column density of the two gas phases is also shown in Fig. 3. The peak column densities are $N_{\mathrm{HI}}=(3.0 \pm 0.1) \times 10^{19} \mathrm{~cm}^{-2}$ and $N_{\mathrm{HI}}=(3.9 \pm 0.2) \times 10^{19} \mathrm{~cm}^{-2}$ for the cold and warm gas phase, respectively. The cold gas phase is only detected in the northern part of the cloud, whereas the warm gas phase extends over the entire cloud.

Figure 2e shows the line widths along the major axis of HVC $297+09+253$. The line widths of the cold and warm component are approximately $5 \mathrm{~km} \mathrm{~s}^{-1}$ and $20 \mathrm{~km} \mathrm{~s}^{-1} F W H M$, respectively, without much variation over the extent of the cloud.

The radial velocity (LSR frame) is plotted in Fig. 2f. The cold gas phase has $v_{\text {LSR }} \approx 253 \mathrm{~km} \mathrm{~s}^{-1}$. The warm component has $v_{\text {LSR }} \approx 253 \mathrm{~km} \mathrm{~s}^{-1}$ close to the position of the cold component and increases up to $256 \mathrm{~km} \mathrm{~s}^{-1}$ towards the southern end of HVC 297+09+253. This velocity gradient will be discussed in Sect. 4.3.

\subsubsection{ATCA data}

The ATCA observations of HVC 297+09+253 were obtained towards the high column density region, the so-called head, of the cloud, where the cold gas phase is found. Figure 4 shows the column density of HVC $297+09+253$ as observed with the ATCA. For comparison, Fig. 2a shows the column density map of HVC $297+09+253$ observed with Parkes superposed by the ATCA observations as white contours.

The total H I mass is determined by

$M_{\mathrm{HI}}=m_{\mathrm{H}} d^{2} \tan ^{2} \varphi \sum_{i} N_{\mathrm{HI}}^{(i)}$ 

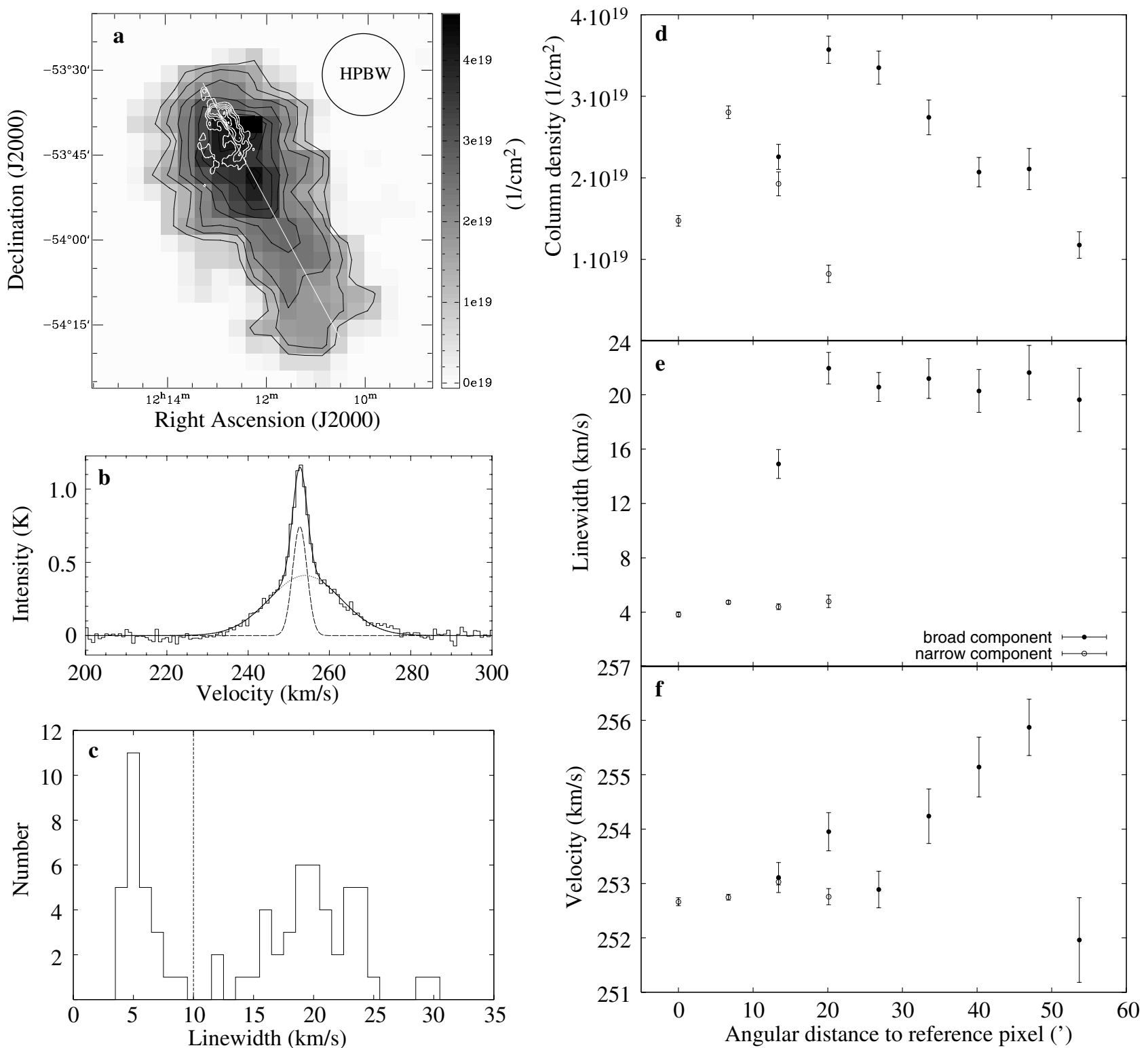

Fig. 2. a) H I column density map of HVC $297+09+253$ observed with the Parkes telescope. The angular resolution of HPBW = 14'. 1 is indicated in the upper right. The black contours range from $1 \times 10^{19}$ to $4 \times 10^{19} \mathrm{~cm}^{-2}$ in steps of $5 \times 10^{18} \mathrm{~cm}^{-2}$. Overlaid are the contour lines (white) of this cloud observed with the ATCA $\left(5 \times 10^{19}\right.$ to $3 \times 10^{20} \mathrm{~cm}^{-2}$ in steps of $\left.5 \times 10^{19} \mathrm{~cm}^{-2}\right)$. The ATCA data cover the northern half of the cloud. The white line indicates the major axis of HVC $297+09+253$. b) The average of all 59 spectra with $T_{\mathrm{B}}>3 \sigma_{\mathrm{rms}}$ observed with Parkes. Apparently, there is a superposition of a narrow and a broad line component. We fitted two Gaussian functions into the spectrum. The sum of the two fits is also shown (solid curve). The resulting fit reproduces the line profile very well. c) The histogram shows the distribution of line widths of HVC 297+09+253. Two separate line components are present. A line width of $\Delta v_{F W H M}=10 \mathrm{~km} \mathrm{~s}^{-1} F W H M$ (dashed line) can be used to separate the two line components. d)-f) Column density, $N_{\mathrm{HI}}$, linewidth, $\Delta v_{F W H M}$, and mean velocity, $v_{\mathrm{LSR}}$, from the two-component Gaussian fits along the major axis of HVC $297+09+253$ (see Fig. 2a). The diagrams demonstrate that the cool and the warm gas phase are partly spatially separated.

where $m_{\mathrm{H}}$ is the mass of a hydrogen atom, $d$ is the distance to the cloud, $\varphi$ is the angular size of a single pixel of the column density map, and $N_{\mathrm{HI}}$ is the column density. The total H I mass detected by the ATCA is $M_{\mathrm{HI}}=1.7 \times 10^{4} M_{\odot}[d / 50 \mathrm{kpc}]^{2}$, while the total mass detected with Parkes is $M_{\mathrm{H} \text { I }}=4.7 \times 10^{4} M_{\odot}[d / 50 \mathrm{kpc}]^{2}$. We determine a lower detection limit $M_{\text {limit }}$ by computing the mass of a point source with a typical line width which generates a $3 \sigma_{\text {rms }}$ (Parkes) and $5 \sigma_{\text {rms }}$ (ATCA) signal in the mass map. For a typical line width of $\Delta v_{F W H M}=4 \mathrm{~km} \mathrm{~s}^{-1}$ we get $M_{\text {limit }}=600 M_{\odot}$ (Parkes) and $M_{\text {limit }}=40 M_{\odot}$ (ATCA). A comparison of the total H I masses obtained for the Parkes and ATCA data for HVC $297+09+253$ reveals that the ATCA detects only $36 \%$ of the mass of HVC $297+09+253$. This result demonstrates that the diffuse extended gaseous phase, constituting a major part of the total H I mass, is missed by the interferometer.

A combination of the single-dish and the interferometer data is nevertheless not meaningful. While the warm gas phase has considerable column densities, the line intensities are quite low due to the large line widths. The rms noise of the ATCA data $\left(\sigma_{\mathrm{rms}} \approx 1.7 \mathrm{~K}\right)$ is significantly larger than typical line intensities of the warm component, which are well below $1 \mathrm{~K}$ (the noise of the Parkes data of $\sigma_{\text {rms }} \approx 0.1 \mathrm{~K}$ is much lower).

The high resolution ATCA data cube reveals a clumpy substructure of HVC 297+09+253 (see Fig. 4). Gaussian functions were fitted to the spectra of all clumps to analyse their physical properties in detail. All clumps in HVC $297+09+253$ have very 


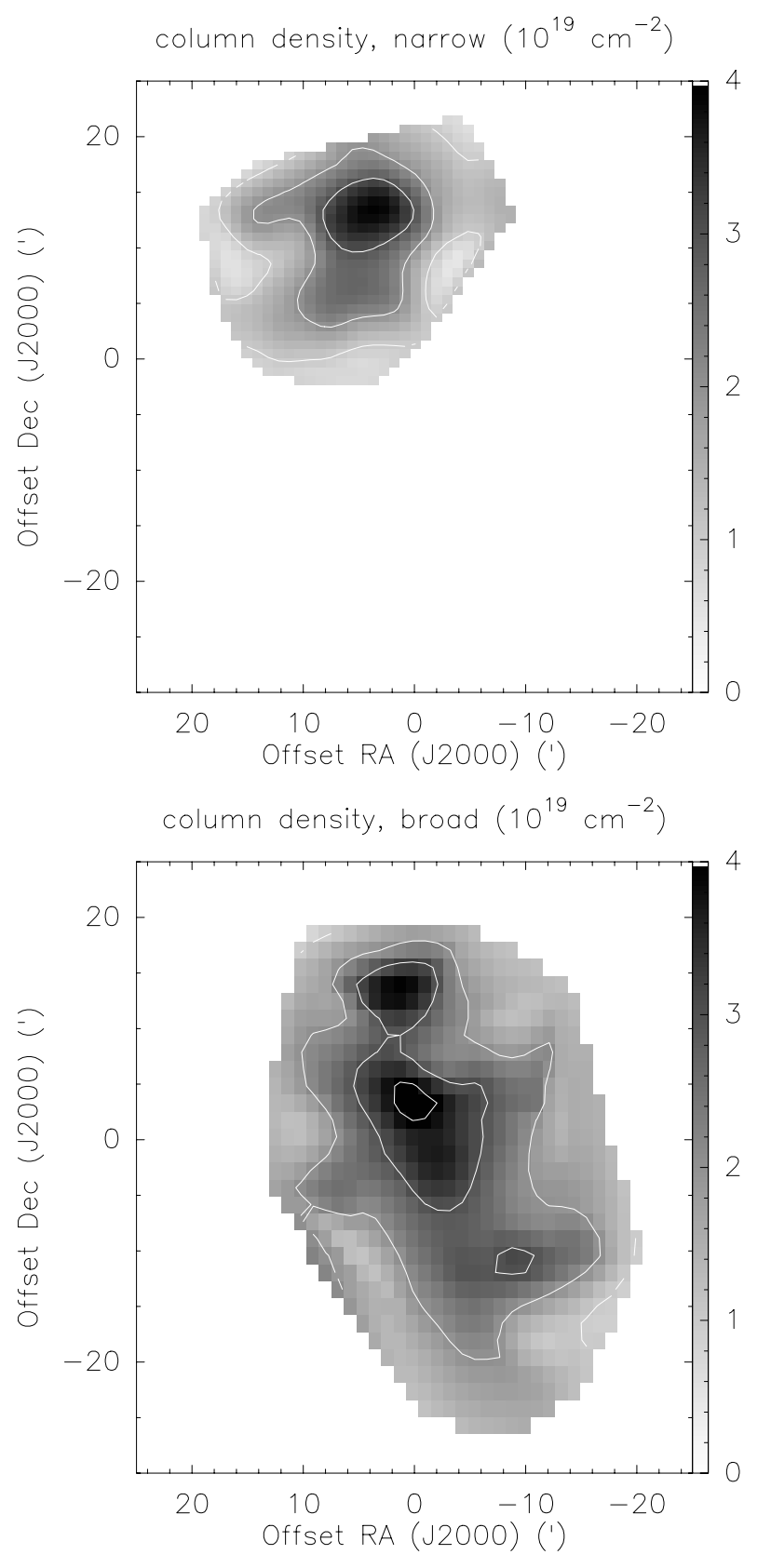

Fig. 3. The spatial distribution of the column densities of both line components of HVC 297+09+253. Upper panel: column density of the narrow line component. Lower panel: column density of the broad component. The contours range from $1 \times 10^{19}$ to $4 \times 10^{19} \mathrm{~cm}^{-2}$ in steps of $1 \times 10^{19} \mathrm{~cm}^{-2}$.

small line widths between 2 and $4 \mathrm{~km} \mathrm{~s}^{-1} F W H M$. The corresponding upper limit for the kinetic temperature is $T_{\text {kin }} \leq 300 \mathrm{~K}$. The clumps have relatively high column densities of $N_{\mathrm{HI}} \approx$ $10^{20} \mathrm{~cm}^{-2}$ and small angular sizes of $\varphi \approx 1^{\prime} \ldots 2^{\prime} F W H M$. The physical parameters are shown in Table 1 .

The position of one exemplary core, clump A, is marked in Fig. 4. Clump A has a column density of $N_{\mathrm{HI}}=(1.3 \pm 0.1) \times$ $10^{20} \mathrm{~cm}^{-2}$. The spectrum of clump A (see Fig. 4) has a very small line width of $\Delta v_{F W H M}=2.3 \pm 0.1 \mathrm{~km} \mathrm{~s}^{-1}$, yielding an upper temperature limit of $T_{\mathrm{D}}=119 \pm 13 \mathrm{~K}$.

The radiative transfer equation for the $21 \mathrm{~cm}$ line emission in the case without background emission is

$T_{\mathrm{B}}=T_{\mathrm{spin}}\left(1-\mathrm{e}^{-\tau}\right)$,
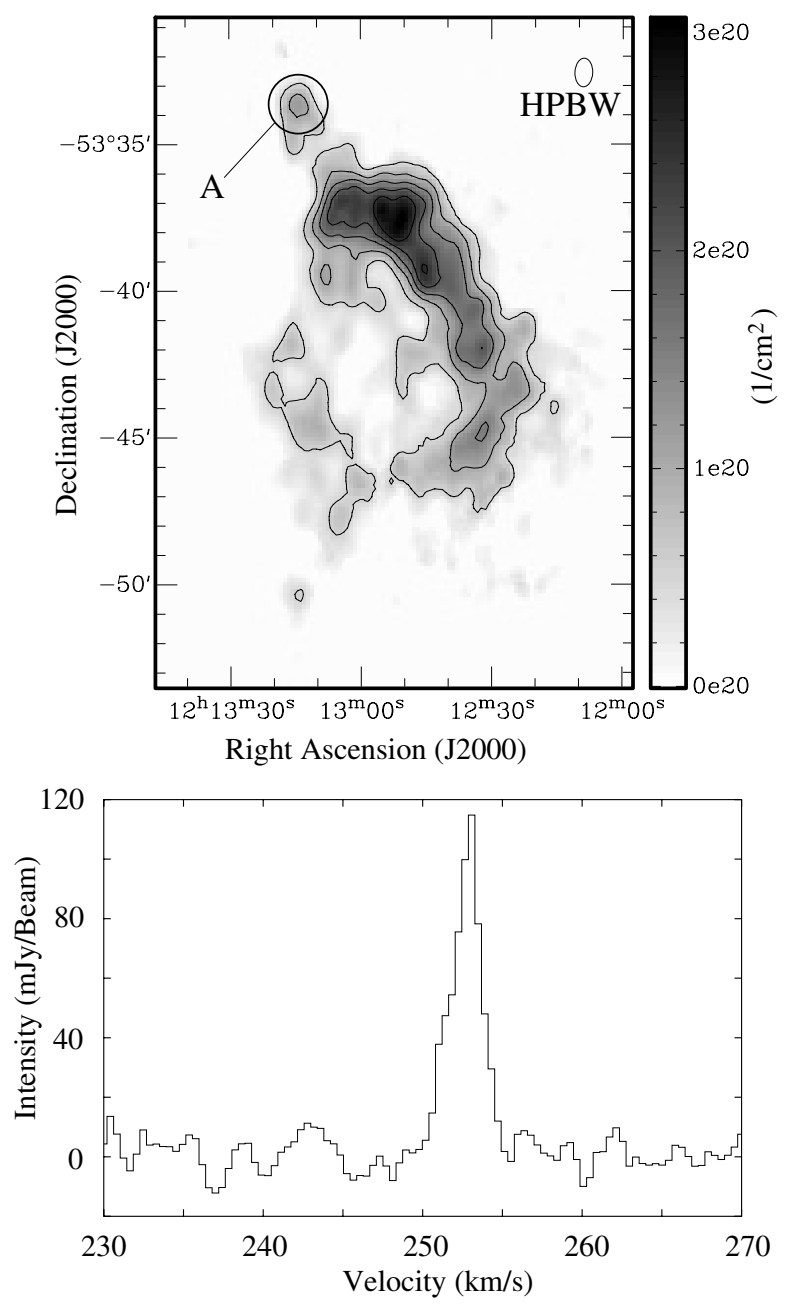

Fig. 4. Upper panel: column density map of the ATCA data cube of HVC 297+09+253. The black circle marks the position of clump A, which is analysed in detail regarding its physical properties. The contours range from $5 \times 10^{19}$ to $3 \times 10^{20} \mathrm{~cm}^{-2}$ in steps of $5 \times 10^{19} \mathrm{~cm}^{-2}$. The ATCA beam of 58.6" $\times 36.2^{\prime \prime}$ HPBW is also shown. Lower panel: A typical spectrum in the direction of clump A of HVC $297+09+253$.

where $T_{\text {spin }}$ is the spin temperature and $\tau$ the optical depth. The equation shows that $T_{\mathrm{B}} \leq T_{\text {spin. }}$. In the case of a thermal excitation it is expected that $T_{\text {spin }} \approx T_{\text {kin. }}$. The measured brightness temperature $T_{\mathrm{B}}$ is therefore a lower temperature limit, leading to a possible temperature range of $T_{\mathrm{B}} \leq T_{\text {kin }} \leq T_{\mathrm{D}}$ of the $\mathrm{HI}$ gas. The brightness temperature for clump $\mathrm{A}$ is $T_{\mathrm{B}}=32 \pm 4 \mathrm{~K}$. The very small line width allows us to constrain the kinetic temperature of this clump to $32 \mathrm{~K} \leq T_{\text {kin }} \leq 120 \mathrm{~K}$.

Furthermore, Eq. (3) allows us to estimate a lower limit for the optical depth of clump A of $\tau \geq 0.31 \pm 0.05$. This is a remarkable result because the approximation $\tau \ll 1$ (which is the common assumption to derive the column density as a linear function of $T_{\mathrm{B}}$ ) is not satisfied anymore. As we have only lower limits for the optical depth, we did not correct the column densities, which accordingly represent lower limits.

The neutral atomic hydrogen density of the condensation can be estimated assuming a spherical symmetry and a constant density $n=\frac{N_{\mathrm{HI}}}{\tan \phi d}$, where $N_{\mathrm{HI}}$ is the column density, $\phi$ is the angular diameter of the clump, and $d$ is the distance to the cloud. Condensation A is sufficiently isolated and circular symmetric to expect that its extent along the line of sight is similar to its apparent diameter in the plane of the sky. The estimated H I 
Table 1. Important physical parameters for all clumps of HVC $297+09+253$. For all distance-dependent parameters a distance of $50 \mathrm{kpc}$ is assumed. The columns give the name of the clump, the coordinates, the velocity in the LSR frame, the line width $\triangle v_{F W H M}$, the lower and upper temperature limits $T_{\min }$ and $T_{\max }$, the $S N R$, the optical depth $\tau$, the column density $N_{\mathrm{HI}}$, the angular diameter $\phi$, the particle density $n$, and the lower $\left(\frac{p}{k_{\mathrm{B}}}\right)_{\min }$ and upper pressure limit $\left(\frac{p}{k_{\mathrm{B}}}\right)_{\max }$ as well as the estimated distances $d$.

\begin{tabular}{|c|c|c|c|c|c|c|c|c|c|c|c|c|c|c|}
\hline Clump & RA & $\overline{\text { Dec }}$ & $\begin{array}{c}v_{\mathrm{LSR}} \\
{\left[\mathrm{km} \mathrm{s}^{-1}\right]}\end{array}$ & $\begin{array}{l}\Delta v_{F W H M} \\
{\left[\mathrm{~km} \mathrm{~s}^{-1}\right]}\end{array}$ & $\begin{array}{c}T_{\max } \\
{[\mathrm{K}]}\end{array}$ & $\begin{array}{l}T_{\min } \\
{[\mathrm{K}]}\end{array}$ & SNR & $\tau_{\min }$ & $\begin{array}{c}N_{\mathrm{HI}} \\
{\left[\mathrm{cm}^{-2}\right]}\end{array}$ & $\begin{array}{c}\phi \\
{\left[{ }^{\prime \prime}\right]}\end{array}$ & $\begin{array}{c}n \\
{\left[\mathrm{~cm}^{-3}\right]}\end{array}$ & $\begin{array}{c}\left(\frac{p}{k}\right)_{\min } \\
{\left[\mathrm{cm}^{-3} \mathrm{~K}\right]}\end{array}$ & $\begin{array}{c}\left(\frac{p}{k}\right)_{\max } \\
{\left[\mathrm{cm}^{-3} \mathrm{~K}\right]}\end{array}$ & $\begin{array}{c}d \\
{[\mathrm{kpc}]}\end{array}$ \\
\hline$\overline{\mathrm{A}}$ & $12^{\mathrm{h}} 13^{\mathrm{m}} 14.5^{\mathrm{s}}$ & $-53^{\circ} 33^{\prime} 32.8^{\prime \prime}$ & 252.8 & 2.3 & 119 & 32 & 16.4 & 0.27 & $1.3 \times 10^{20}$ & 88 & 2.1 & 67 & 256 & 27 \\
\hline M & $12^{\mathrm{h}} 12^{\mathrm{m}} 35.9^{\mathrm{s}}$ & $-53^{\circ} 45^{\prime} 18.0^{\prime \prime}$ & 253.3 & 4.2 & 379 & 21 & 10.8 & 0.06 & $1.4 \times 10^{20}$ & 96 & 2.2 & 44 & 821 & \\
\hline $\mathrm{O}$ & $12^{\mathrm{h}} 12^{\mathrm{m}} 30.5^{\mathrm{s}}$ & $-53^{\circ} 43^{\prime} 26.0^{\prime \prime}$ & 252.6 & 3.3 & 237 & 20 & 10.2 & 0.08 & $9.7 \times 10^{19}$ & 112 & 1.3 & 26 & 302 & \\
\hline I & $12^{\mathrm{h}} 12^{\mathrm{m}} 36.8^{\mathrm{s}}$ & $-53^{\circ} 42^{\prime} 14.0^{\prime \prime}$ & 253.7 & 3.9 & 332 & 26 & 13.2 & 0.08 & $1.5 \times 10^{20}$ & 80 & 2.8 & 75 & 928 & \\
\hline K & $12^{\mathrm{h}} 12^{\mathrm{m}} 38.6^{\mathrm{s}}$ & $-53^{\circ} 38^{\prime} 45.9^{\prime \prime}$ & 252.9 & 3.4 & 249 & 29 & 14.5 & 0.11 & $1.7 \times 10^{20}$ & 112 & 2.2 & 62 & 544 & 19 \\
\hline $\mathrm{N}$ & $12^{\mathrm{h}} 12^{\mathrm{m}} 34.1^{\mathrm{s}}$ & $-53^{\circ} 40^{\prime} 30.0^{\prime \prime}$ & 253.4 & 3.9 & 325 & 24 & 12.1 & 0.07 & $1.5 \times 10^{20}$ & 104 & 2.1 & 56 & 677 & \\
\hline $\mathrm{C}$ & $12^{\mathrm{h}} 13^{\mathrm{m}} 15.4^{\mathrm{s}}$ & $-53^{\circ} 34^{\prime} 36.9^{\prime \prime}$ & 253.7 & 2.4 & 127 & 22 & 11.3 & 0.18 & $9.7 \times 10^{19}$ & 136 & 1.0 & 20 & 133 & 29 \\
\hline $\mathrm{D}$ & $12^{\mathrm{h}} 13^{\mathrm{m}} 16.4^{\mathrm{s}}$ & $-53^{\circ} 41^{\prime} 41.3^{\prime \prime}$ & 252.7 & 2.7 & 159 & 20 & 10.3 & 0.13 & $9.1 \times 10^{19}$ & 96 & 1.4 & 23 & 222 & \\
\hline $\mathrm{F}$ & $12^{\mathrm{h}} 13^{\mathrm{m}} 20.1^{\mathrm{s}}$ & $-53^{\circ} 43^{\prime} 25.2^{\prime \prime}$ & 252.6 & 3.2 & 216 & 16 & 8.2 & 0.07 & $8.7 \times 10^{19}$ & 80 & 1.6 & 19 & 345 & \\
\hline G & $12^{\mathrm{h}} 13^{\mathrm{m}} 12.0^{\mathrm{s}}$ & $-53^{\circ} 44^{\prime} 29.4^{\prime \prime}$ & 252.2 & 3.1 & 204 & 19 & 9.4 & 0.09 & $1.0 \times 10^{20}$ & 88 & 1.8 & 26 & 358 & \\
\hline $\mathrm{P}$ & $12^{\mathrm{h}} 13^{\mathrm{m}} 10.2^{\mathrm{s}}$ & $-53^{\circ} 46^{\prime} 21.4^{\prime \prime}$ & 252.1 & 2.0 & 83 & 16 & 8.0 & 0.19 & $5.6 \times 10^{19}$ & 88 & 0.9 & 11 & 77 & 37 \\
\hline Q & $12^{\mathrm{h}} 13^{\mathrm{m}} 08.3^{\mathrm{s}}$ & $-53^{\circ} 39^{\prime} 25.5^{\prime \prime}$ & 252.5 & 2.4 & 122 & 27 & 13.8 & 0.22 & $1.3 \times 10^{20}$ & 144 & 1.3 & 31 & 158 & \\
\hline $\mathrm{H}$ & $12^{\mathrm{h}} 12^{\mathrm{m}} 48.5^{\mathrm{s}}$ & $-53^{\circ} 41^{\prime} 57.9^{\prime \prime}$ & 253.5 & 2.5 & 131 & 19 & 9.8 & 0.15 & $8.5 \times 10^{19}$ & 88 & 1.4 & 24 & 185 & \\
\hline $\mathrm{L}$ & $12^{\mathrm{h}} 12^{\mathrm{m}} 55.7^{\mathrm{s}}$ & $-53^{\circ} 41^{\prime} 29.8^{\prime \prime}$ & 253.4 & 2.8 & 176 & 16 & 8.1 & 0.09 & $8.3 \times 10^{19}$ & 80 & 1.5 & 19 & 267 & \\
\hline $\mathrm{R}$ & $12^{\mathrm{h}} 13^{\mathrm{m}} 13.0^{\mathrm{s}}$ & $-53^{\circ} 50^{\prime} 21.1^{\prime \prime}$ & 252.4 & 2.4 & 130 & 14 & 7.0 & 0.11 & $6.2 \times 10^{19}$ & 64 & 1.4 & 13 & 185 & \\
\hline S & $12^{\mathrm{h}} 13^{\mathrm{m}} 03.9^{\mathrm{s}}$ & $-53^{\circ} 47^{\prime} 41.5^{\prime \prime}$ & 252.8 & 3.4 & 245 & 14 & 7.1 & 0.06 & $8.2 \times 10^{19}$ & 96 & 1.3 & 15 & 307 & \\
\hline $\mathrm{T}$ & $12^{\mathrm{h}} 12^{\mathrm{m}} 49.4^{\mathrm{s}}$ & $-53^{\circ} 37^{\prime} 33.4^{\prime \prime}$ & 252.8 & 3.5 & 262 & 49 & 24.6 & 0.19 & $3.0 \times 10^{20}$ & 152 & 2.9 & 138 & 752 & 22 \\
\hline
\end{tabular}

volume density for condensation A is $n=2.1 \mathrm{~cm}^{-3}[d / 50 \mathrm{kpc}]^{-1}$. Table 1 compiles the estimated densities of all condensations of HVC $297+09+253$. Note, however, that most of them are embedded in an enveloping medium making the estimates less reliable. Furthermore we cannot rule out that the cold clumps contain substructure at sub-arcmin scale which is not resolved with our ATCA data. Consequently, the filling factor may be less than unity. Potential gaps between the dense clumps may be filled with warm neutral gas, but we can rule out the presence of molecular or highly ionised gas. Even the densest clumps have densities which are far too low to allow for the formation of molecules. There is not even a reliable indication of the existence of dust in HVCs (e.g. Wakker \& Boulanger 1986). Highly ionised gas also cannot exist in the inner parts of HVCs because they are shielded against the extragalactic radiation field by the surrounding warm neutral medium. For example, Sternberg et al. (2002) perform hydrostatic simulations of CHVCs embedded in an ionised intergalactic medium. In their model the ionisation rate of hydrogen drops by several orders of magnitude from $1 \times 10^{-14} \mathrm{~s}^{-1}$ to $1 \times 10^{-17} \mathrm{~s}^{-1}$ from the outer edge to the centre of the cloud. In addition, the diffusion of hot plasma from the surrounding medium into the neutral cloud will be suppressed by a magnetic field in the intergalactic medium due to a magnetic barrier of increased field strength building up at the interface of the HVC (Konz et al. 2002).

The pressure of the condensations can be estimated using the ideal gas equation,

$\frac{p}{k_{\mathrm{B}}}=\frac{N_{\mathrm{HI}}}{d \tan \phi} T$

where $p$ is the pressure and $k_{\mathrm{B}}$ the Boltzmann constant. We derive a relatively narrow range of allowed pressures for condensation $\mathrm{A}$ of $\left(p / k_{\mathrm{B}}\right)_{\min }=67 \pm 15 \mathrm{~K} \mathrm{~cm}^{-3}[d / 50 \mathrm{kpc}]^{-1}$ and $\left(p / k_{\mathrm{B}}\right)_{\max }=256 \pm 53 \mathrm{~K} \mathrm{~cm}^{-3}[d / 50 \mathrm{kpc}]^{-1}$. The distance of HVC $297+09+253$ is discussed in Sects. 4.2 and 4.3. Table 1 shows the distance-dependent parameters size, mass, particle number, density, and pressure for all clumps of HVC $297+09+253$.

\subsection{HVC $291+26+195$}

\subsubsection{Parkes data}

Figure 5a shows the column density map of HVC 291+26+195. Apparently, the Parkes beam of 14!'1 HPBW does not resolve the HVC. The investigation of the line profiles reveals that there is only one line component in the spectra of this cloud. In Fig. 5b we plotted the average of all 12 spectra of HVC 291+26+195. The line width of the component is about $5 \mathrm{~km} \mathrm{~s}^{-1} F W H M$. The resulting upper temperature limit is $T_{\max }=640 \mathrm{~K}$. The data show no evidence for a warm gas phase. The peak column density is $N_{\mathrm{HI}}=(1.3 \pm 0.1) \times 10^{19} \mathrm{~cm}^{-2}$. The resulting parameters from the Gaussian fits are similar to the results for the cold component of HVC $297+09+253$.

\subsubsection{ATCA data}

Figure 5c shows a column density map of HVC 291+26+195 observed with the ATCA. The column density distribution of the HVC is elongated containing several unresolved concentrations. The main filament is accompanied by a few small clouds. The peak column densities of the individual clumps range from $3 \times$ $10^{19} \mathrm{~cm}^{-2}$ to $1 \times 10^{20} \mathrm{~cm}^{-2}$.

The total HI mass of HVC $291+26+195$ is $M_{\mathrm{HI}}=5.9 \times$ $10^{3} M_{\odot}\left[d^{2} / 50 \mathrm{kpc}\right]$ and $M_{\mathrm{H} \mathrm{I}}=5.6 \times 10^{3} M_{\odot}\left[d^{2} / 50 \mathrm{kpc}\right]$, measured with Parkes and ATCA respectively. The ATCA detects $95 \%$ of the mass which was measured with Parkes, confirming that not much diffuse gas is present in HVC $291+26+195$.

The HI lines of HVC $291+26+195$ are quite narrow $\left(\Delta v_{F W H M}=2 \ldots 4 \mathrm{~km} \mathrm{~s}^{-1}\right)$. As an example Fig. 5d shows the spectrum of clump $\mathrm{K}$ that has a line width of $\Delta v_{F W H M}=$ $3.5 \mathrm{~km} \mathrm{~s}^{-1}$. The line widths of all clumps are between $2 \ldots 4 \mathrm{~km} \mathrm{~s}^{-1}$. Assuming a distance of $d=50 \mathrm{kpc}$ (see 


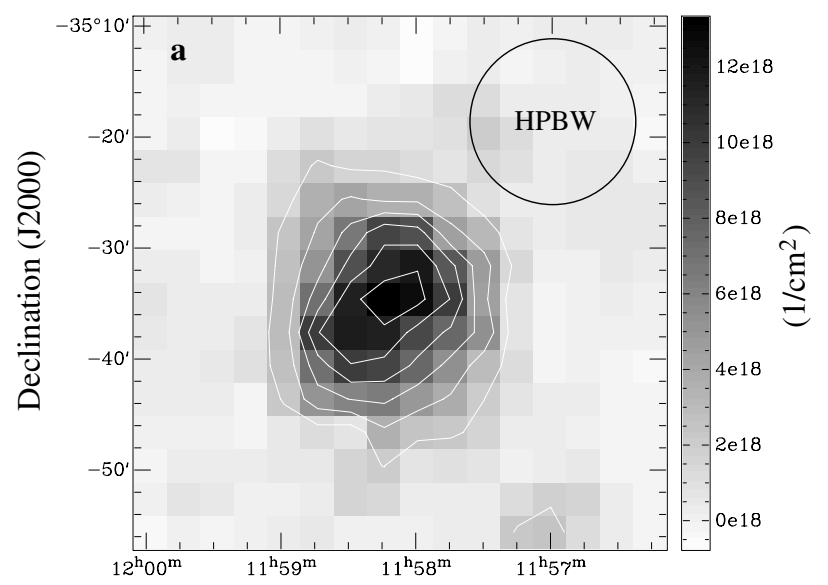

Right Ascension (J2000)

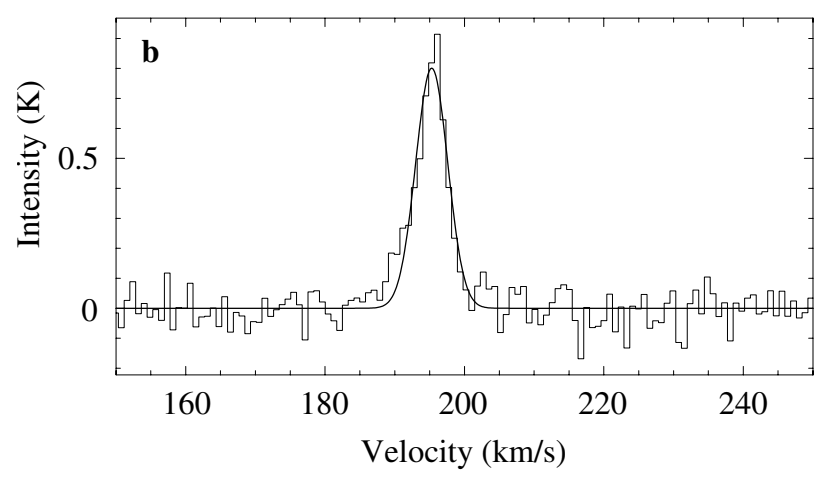

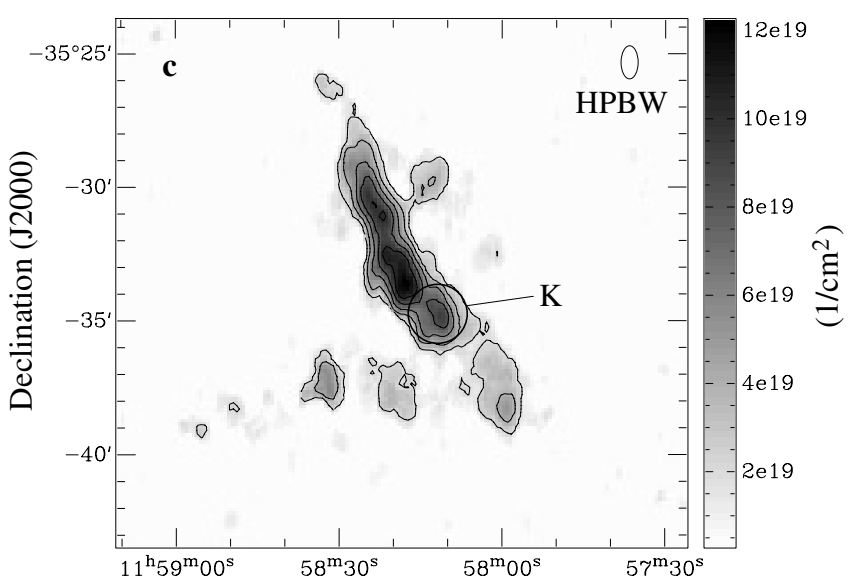

Right Ascension (J2000)

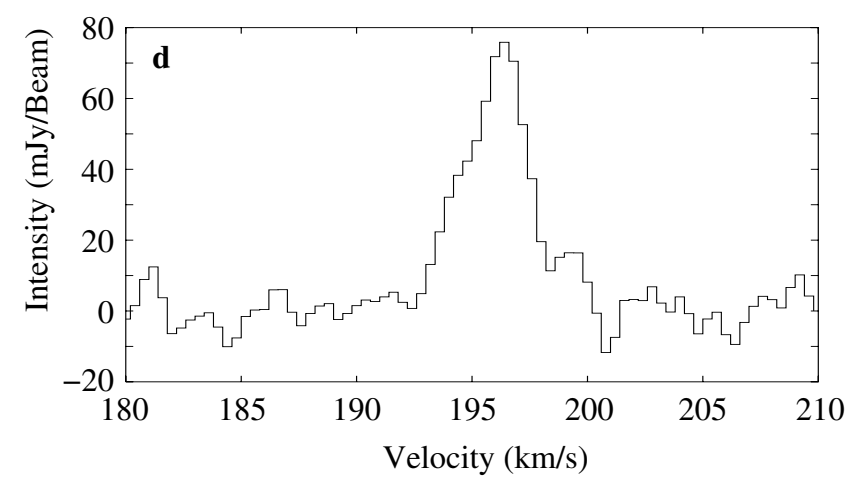

Fig. 5. a) H I column density map of HVC $291+26+195$ observed with Parkes. The contours range from $2 \times 10^{18}$ to $1.2 \times 10^{19} \mathrm{~cm}^{-2}$ in steps of $2 \times 10^{18} \mathrm{~cm}^{-2}$. b) Average spectrum of all 12 positions in the direction of HVC $291+26+195$ in which the spectral line was detected with at least $3 \sigma$. The Gaussian fit (solid line) indicates that only a single line component is present. c) Column density map of HVC $291+26+195$ observed with the ATCA. The contours range from $2 \times 10^{19}$ to $1.2 \times 10^{20} \mathrm{~cm}^{-2}$ in steps of $2 \times 10^{19} \mathrm{~cm}^{-2}$. The ATCA beam of $74.6^{\prime \prime} \times 38.4^{\prime \prime} \mathrm{HPBW}$ is also shown. d) A typical spectrum of clump K.

Sects. 4.2 and 4.3), the upper limit for the pressure is $\left(p / k_{\mathrm{B}}\right)_{\max }=378 \pm 67 \mathrm{~K} \mathrm{~cm}^{-3}[d / 50 \mathrm{kpc}]^{-1}$ for clump $\mathrm{K}$ and $\left(p / k_{\mathrm{B}}\right)_{\max }=154 \pm 43 \mathrm{~K} \mathrm{~cm}^{-3}[d / 50 \mathrm{kpc}]^{-1}$ for clump $\mathrm{N}$ of HVC $291+26+195$. The important parameters of all investigated clumps of HVC $291+26+195$ are listed in Table 2 .

In summary, the ATCA observations of HVC 291+26+195 reveal, as expected, only a cold gas phase having physical properties similar to the cold phase of HVC $297+09+253$.

\section{Discussion}

\subsection{Evidence for ram-pressure interaction}

The HI data of HVC 297+09+253 show a distinct head-tail structure. The high column density region, the head of the cloud, contains both a narrow and a broad component. In the northernmost part of the CHVC only a narrow line component is detected, while there is solely a broad component in the tail (see Sect. 3.1).

The observed morphology suggests a ram-pressure interaction with an ambient medium, where the warm gas phase is stripped off the CHVC due to friction forces (Brüns et al. 2000, 2001). The clumps may have formed due to compression of the gas at the leading edge of an CHVC and local density fluctuations in combination with cooling processes.

The existence of a two-component gas structure is widely observed among HVCs and CHVCs (Cram \& Giovanelli 1976; Wolfire et al. 1995; Burton et al. 2001; de Heij et al. 2002;
Braun \& Burton 1999; Westmeier et al. 2005). The 2D hydrostatic simulations of CHVCs by Sternberg et al. (2002) are consistent with these observations. Neutral atomic hydrogen clouds can accordingly show a stable multiphase structure in an environment with conditions comparable to those in the outer Galactic halo. The hydrodynamical simulations of Quilis \& Moore (2001) and Vieser \& Hensler (2001) of HVCs moving through a diffuse medium show that the lowest temperatures of interacting HVCs are found in the head of the clouds whereas higher temperatures occur in the tail of HVCs where the material was stripped off the main body of the cloud. Konz et al. (2002) show in their hydrodynamical simulations that the cold gas in the head of the cloud remains unaffected by the stripping process. This is qualitatively consistent with our observations of HVC $297+09+253$. The smallest line widths of $\Delta v_{F W H M} \approx 2 \mathrm{~km} \mathrm{~s}^{-1}$ (indicating the lowest temperatures) are located in the head of HVC $297+09+253$ while the broadest lines of about $\Delta v_{F W H M}=20 \mathrm{~km} \mathrm{~s}^{-1}$ are observed in the tail.

The tail of HVC $297+09+253$ shows higher radial velocities than the high column density head (Fig. 2f). Brüns et al. (2000) analysed 252 HVCs. $20 \%$ of the clouds show a head-tail structure combined with a gradient in column density and in velocity along the major axis of the clouds. But none of the HVCs in this sample shows an increasing velocity in the direction of the tail.

Part of this gradient can be explained as a projection effect from the solar velocity vector. The gradient decreases slightly from $\Delta v_{\mathrm{LSR}}=3 \mathrm{~km} \mathrm{~s}^{-1}$ to about $\Delta v_{\mathrm{GSR}}=2.5 \mathrm{~km} \mathrm{~s}^{-1}$ when 
Table 2. Important physical parameters for all clumps of HVC $291+26+195$. For all distance-dependent parameters a distance of 50 kpc is assumed. The columns give the name of the clump, the coordinates, the velocity in the LSR frame, the line width $\triangle v_{F W H M}$, the lower and upper temperature limits $T_{\min }$ and $T_{\max }$, the $S N R$, the optical depth $\tau$, the column density $N_{\mathrm{HI}}$, the angular diameter $\phi$, the particle density $n$, and the lower $\left(\frac{p}{k_{\mathrm{B}}}\right)_{\min }$ and upper pressure limit $\left(\frac{p}{k_{\mathrm{B}}}\right)_{\max }$ as well as the estimated distances $d$.

\begin{tabular}{|c|c|c|c|c|c|c|c|c|c|c|c|c|c|c|}
\hline Clump & RA & Dec & $\begin{array}{c}v_{\mathrm{LSR}} \\
{\left[\mathrm{km} \mathrm{s}^{-1}\right]}\end{array}$ & $\begin{array}{c}\Delta v_{F W H M} \\
{\left[\mathrm{~km} \mathrm{~s}^{-1}\right]}\end{array}$ & $\begin{array}{c}T_{\max } \\
{[\mathrm{K}]}\end{array}$ & $\begin{array}{l}T_{\min } \\
{[\mathrm{K}]}\end{array}$ & SNR & $\tau_{\min }$ & $\begin{array}{c}N_{\mathrm{HI}} \\
{\left[\mathrm{cm}^{-2}\right]}\end{array}$ & $\begin{array}{c}\phi \\
{\left[{ }^{\prime \prime}\right]}\end{array}$ & $\begin{array}{c}n \\
{\left[\mathrm{~cm}^{-3}\right]}\end{array}$ & $\begin{array}{c}\left(\frac{p}{k}\right)_{\min } \\
{\left[\mathrm{cm}^{-3} \mathrm{~K}\right]}\end{array}$ & $\begin{array}{c}\left(\frac{p}{k}\right)_{\max } \\
{\left[\mathrm{cm}^{-3} \mathrm{~K}\right]}\end{array}$ & $\begin{array}{c}d \\
{[\mathrm{kpc}]}\end{array}$ \\
\hline A & $11^{\mathrm{h}} 58^{\mathrm{m}} 31.7^{\mathrm{s}}$ & $-35^{\circ} 37^{\prime} 15.7^{\prime \prime}$ & 192.1 & 3.4 & 6.9 & 251 & 10 & 0.04 & $6.0 \times 10^{19}$ & 72 & 1.2 & 12 & 307 & 16 \\
\hline B & $11^{\mathrm{h}} 58^{\mathrm{m}} 48.8^{\mathrm{s}}$ & $-35^{\circ} 38^{\prime} 26.9^{\prime \prime}$ & 192.0 & 2.5 & 5.7 & 137 & 8 & 0.06 & $3.8 \times 10^{19}$ & 80 & 0.7 & 6 & 96 & \\
\hline $\mathrm{C}$ & $11^{\mathrm{h}} 58^{\mathrm{m}} 35.7^{\mathrm{s}}$ & $-35^{\circ} 37^{\prime} 39.5^{\prime \prime}$ & 191.2 & 3.0 & 5.3 & 195 & 8 & 0.04 & $3.8 \times 10^{19}$ & 88 & 0.6 & 5 & 124 & \\
\hline D & $11^{\mathrm{h}} 58^{\mathrm{m}} 17.3^{\mathrm{s}}$ & $-35^{\circ} 38^{\prime} 19.8^{\prime \prime}$ & 194.9 & 2.3 & 6.0 & 112 & 9 & 0.08 & $3.6 \times 10^{19}$ & 96 & 0.6 & 5 & 63 & 35 \\
\hline $\mathrm{E}$ & $11^{\mathrm{h}} 58^{\mathrm{m}} 21.2^{\mathrm{s}}$ & $-35^{\circ} 37^{\prime} 47.9^{\prime \prime}$ & 194.5 & 3.5 & 5.2 & 272 & 8 & 0.03 & $4.0 \times 10^{19}$ & 120 & 0.5 & 4 & 134 & \\
\hline $\mathrm{F}$ & $11^{\mathrm{h}} 58^{\mathrm{m}} 18.6^{\mathrm{s}}$ & $-35^{\circ} 37^{\prime} 07.9^{\prime \prime}$ & 194.0 & 2.7 & 5.2 & 155 & 8 & 0.05 & $2.9 \times 10^{19}$ & 112 & 0.4 & 3 & 59 & \\
\hline G & $11^{\mathrm{h}} 58^{\mathrm{m}} 54.7^{\mathrm{s}}$ & $-35^{\circ} 38^{\prime} 58.5^{\prime \prime}$ & 193.7 & 3.8 & 4.7 & 310 & 7 & 0.02 & $5.3 \times 10^{19}$ & 54 & 1.4 & 10 & 445 & \\
\hline $\mathrm{H}$ & $11^{\mathrm{h}} 58^{\mathrm{m}} 33.0^{\mathrm{s}}$ & $-35^{\circ} 26^{\prime} 02.8^{\prime \prime}$ & 195.9 & 2.5 & 5.3 & 136 & 8 & 0.06 & $3.9 \times 10^{19}$ & 88 & 0.7 & 5 & 90 & \\
\hline I & $11^{\mathrm{h}} 58^{\mathrm{m}} 12.0^{\mathrm{s}}$ & $-35^{\circ} 29^{\prime} 39.7^{\prime \prime}$ & 196.0 & 2.8 & 6.7 & 173 & 10 & 0.06 & $4.4 \times 10^{19}$ & 104 & 0.6 & 6 & 107 & \\
\hline $\mathrm{J}$ & $11^{\mathrm{h}} 58^{\mathrm{m}} 03.5^{\mathrm{s}}$ & $-35^{\circ} 32^{\prime} 35.9^{\prime \prime}$ & 195.7 & 1.4 & 4.9 & 45 & 7 & 0.16 & $1.7 \times 10^{19}$ & 96 & 0.3 & 2 & 12 & \\
\hline $\mathrm{K}$ & $11^{\mathrm{h}} 58^{\mathrm{m}} 10.1^{\mathrm{s}}$ & $-35^{\circ} 34^{\prime} 60.0^{\prime \prime}$ & 196.1 & 3.5 & 10.8 & 272 & 16 & 0.06 & $9.8 \times 10^{19}$ & 104 & 1.4 & 22 & 378 & 13 \\
\hline $\mathrm{L}$ & $11^{\mathrm{h}} 58^{\mathrm{m}} 02.8^{\mathrm{s}}$ & $-35^{\circ} 36^{\prime} 35.9^{\prime \prime}$ & 196.2 & 2.5 & 7.3 & 136 & 11 & 0.08 & $4.3 \times 10^{19}$ & 96 & 0.7 & 7 & 91 & \\
\hline M & $11^{\mathrm{h}} 57^{\mathrm{m}} 59.6^{\mathrm{s}}$ & $-35^{\circ} 38^{\prime} 11.7^{\prime \prime}$ & 197.3 & 2.3 & 8.4 & 112 & 12 & 0.11 & $5.5 \times 10^{19}$ & 120 & 0.7 & 8 & 76 & \\
\hline $\mathrm{N}$ & $11^{\mathrm{h}} 58^{\mathrm{m}} 27.8^{\mathrm{s}}$ & $-35^{\circ} 29^{\prime} 15.5^{\prime \prime}$ & 195.3 & 2.2 & 10.0 & 106 & 15 & 0.14 & $6.3 \times 10^{19}$ & 64 & 1.4 & 21 & 154 & 25 \\
\hline $\mathrm{O}$ & $11^{\mathrm{h}} 58^{\mathrm{m}} 23.8^{\mathrm{s}}$ & $-35^{\circ} 30^{\prime} 35.7^{\prime \prime}$ & 195.4 & 3.0 & 13.6 & 198 & 20 & 0.10 & $1.1 \times 10^{20}$ & 104 & 1.6 & 32 & 321 & \\
\hline $\mathrm{P}$ & $11^{\mathrm{h}} 58^{\mathrm{m}} 21.2^{\mathrm{s}}$ & $-35^{\circ} 32^{\prime} 19.9^{\prime \prime}$ & 195.3 & 3.1 & 13.4 & 212 & 20 & 0.09 & $1.1 \times 10^{20}$ & 64 & 2.5 & 49 & 532 & \\
\hline Q & $11^{\mathrm{h}} 58^{\mathrm{m}} 18.6^{\mathrm{s}}$ & $-35^{\circ} 33^{\prime} 48.0^{\prime \prime}$ & 195.2 & 3.2 & 13.2 & 227 & 19 & 0.08 & $1.2 \times 10^{20}$ & 64 & 2.8 & 54 & 634 & \\
\hline
\end{tabular}

transforming into the Galactic standard-of-rest frame. The velocity vector of the HVC produces a similar effect. If we assume that the velocity is produced by a pure projection effect we would get extremely high spatial velocities of the order of $>500 \mathrm{~km} \mathrm{~s}^{-1}$, which is significantly larger than the maximum radial velocities observed for HVCs around the Milky Way.

The apparent contradiction is resolved by considering a slightly varying orientation of the velocity vector over the extent the tail. If the total velocity of the HVC is for instance $200 \mathrm{~km} \mathrm{~s}^{-1}$, a twist of only one degree would be sufficient to explain the observed gradient. A slight deflection of the stripped matter in the tail could have several reasons like an intrinsic velocity of the ambient medium, e.g. a Galactic wind or turbulence in the hot halo of the Milky Way. Moreover, the Galactic magnetic field could influence the gas in the tail. Konz et al. (2002) performed magnetohydrodynamical simulations of interacting HVCs and concluded that magnetic fields play an important role in stabilising the head while forming a tail.

The cloud HVC 291+26+195 shows cold compact clumps with very small line widths comparable to the head of HVC $297+09+253$, but no diffuse component. This might be interpreted as a cloud in a later stage of interaction with the ambient medium, where the diffuse warm component was already stripped off and evaporated within the hot gas phase of the Galactic halo (Quilis \& Moore 2001) while the cold clumps are still neutral and observable in $\mathrm{H}$ I emission.

\subsection{Distance estimate for the two CHVCs}

In Sect. 3.1.2, we estimated the pressure of the cold clumps of HVC 297+09+253 and HVC 291+26+195, assuming an ideal gas, a spherically-symmetric cloud, and a constant particle density. The very small line widths allow to constrain the kinetic temperature of the clumps and, thus, to estimate upper and lower pressure limits for all clumps in the two CHVCs (see Tables 1 and 2) under the above assumptions.
We compare the pressure of some of the clumps in the two CHVCs as a function of distance with the pressure of the surrounding medium derived from the Milky Way model of Kalberla (2003). Figure 6 shows the results for two exemplary clumps of HVC $297+09+253$ and HVC 291+26+195.

The pressure of the clumps should be at least as high as the pressure of the surrounding medium. The intersection point of the pressure curves for the Milky Way gas and the clumps therefore provides a distance estimate. With the mentioned assumptions the corresponding distance estimates for HVC $297+09+253$ and HVC 291+26+195 are in the range of $d \approx 10 \ldots 60 \mathrm{kpc}$ (Tables 1 and 2 ).

One should keep in mind, though, that the distribution of the pressure of the circum-/intergalactic medium is not well constrained by observations. There is observational evidence from absorption line measurements that the thermal pressure could be significant even at larger distances of several hundred kpc from the Galaxy (Sembach et al. 2003). Results from X-ray absorption data by Rasmussen et al. (2003) indicate a substantial value of $P / k_{\mathrm{B}} \approx 100 \mathrm{~cm}^{-3} \mathrm{~K}$ beyond $100 \mathrm{kpc}$ from the Galaxy. A higher value of the pressure would shift the pressure curve of Kalberla (2003) slightly upwards. On the other hand, our calculated values for the internal pressure of some of the clumps are only lower limits because of their significant optical depth for which we cannot correct. Deviations from the previous assumptions (Sect. 3.1.2) add additional uncertainties to the calaculated parameters. Consequently, the determined distances should be considered rough estimates only.

\subsection{Evidence for an association of both CHVCs with the Leading Arm}

Figure 1 shows a column density map of the Magellanic System (Brüns et al. 2005), including the Small and the Large Magellanic Cloud (SMC and LMC), the Magellanic Bridge, which connects the two Magellanic Clouds, the Magellanic 

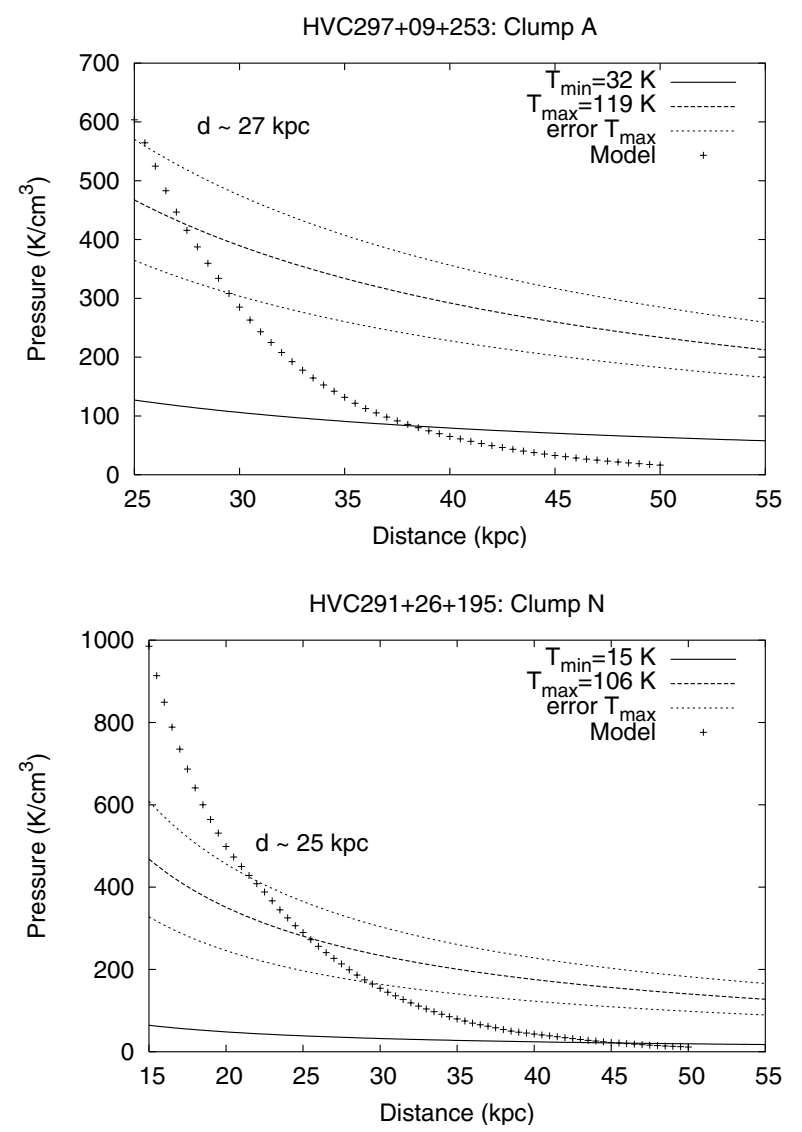

Fig. 6. Pressure variation as a function of distance and statistical error curves (dotted lines) for two clumps of HVC 297+09+253 and HVC $291+26+195$. Furthermore, the pressure variation of the Milky Way gas (crosses) derived from a model developed by Kalberla (2003) is shown. Upper panel: clump A of HVC 297+09+253. Lower panel: clump N of HVC $291+26+195$.

Stream (MS), and the Leading Arm (LA). These features were likely generated in an interaction between the Magellanic Clouds with the Milky Way (Yoshizawa \& Noguchi 2003). The LA consists of many clumpy filaments (Putman et al. 1998) and shows a two-component gas structure (Brüns et al. 2005), comparable to the results derived for HVC 297+09+253 and HVC 291+26+195.

The LA can be divided into three parts, the LAI, II, and III. In the south-eastern part of LA II the observed velocities are between $265 \ldots 300 \mathrm{~km} \mathrm{~s}^{-1}$ in the LSR frame. Thus, HVC $297+09+253$ deviates by only $10 \ldots 15 \mathrm{~km} \mathrm{~s}^{-1}$ from the velocities of this region, which is in good agreement within the velocity dispersion of the gas in LA II of $10 \mathrm{~km} \mathrm{~s}^{-1}$. HVC 291+26+195 lies in the velocity range of the northern part of the LA II $\left(190 \ldots 275 \mathrm{~km} \mathrm{~s}^{-1}\right)$. Numerical simulations of the Magellanic System, e.g. Yoshizawa \& Noguchi (2003), predict distances for the gas in the Leading Arm in the range $30 \ldots 60 \mathrm{kpc}$. This distance range is consistent with the results of the distance estimation for the two clouds described in Sect. 4.2.

The velocity vector of the LMC observed by van der Marel et al. (2002) indicates that the orbit of the Large Magellanic Cloud is in the direction of increasing Galactic latitude. This is consistent with the observed orientation of the tail of HVC 297+09+253, which is perpendicular to the Galactic plane and pointing towards the LMC.

The proximity of the two clouds to the Leading Arm, the comparable velocities, the orientation of the tail and the distance estimates make an association with the Magellanic System likely, implying a distance range of $10 \mathrm{kpc} \leq d \leq 60 \mathrm{kpc}$ for the two HVCs.

\section{Summary and outlook}

We analysed single-dish (Parkes) and interferometer (ATCA) data of two compact high-velocity clouds located in the vicinity of the Leading Arm of the Magellanic System. The observations with both telescopes allow us to investigate the total H I mass and the extended structure as well as the small-scale structure within the clouds.

The analysis of HVC $297+09+253$ reveals that the cloud has a two-component gas structure. The cold and the warm gas phase are partly spatially separated. Cold compact clumps are embedded in a diffuse warm $\mathrm{H}$ I gas. Both the gradient in line width and in column density as well as the morphological asymmetry show that this cloud reveals a head-tail structure. The presence of the cold clumps and the head-tail structure can be explained by an interaction of this cloud with an ambient medium. All clumps show very narrow lines of $\Delta v_{F W H M}<4 \mathrm{~km} \mathrm{~s}^{-1}$, allowing us to constrain physical parameters like the temperature and the pressure of the gas.

In the case of HVC $297+09+253$ we also find cold compact clumps with very narrow lines but no diffuse, warm gas. Possibly, these clumps were also generated in an interaction with an ambient medium. The diffuse gas possibly was already stripped off the cold core due to friction forces.

We presented a method to estimate distances for the two CHVCs by comparing the pressure of the clumps in the CHVCs as a function of distance with the pressure of the surrounding Milky Way gas. The distances estimated for all clumps are of the order of $10 \ldots 60 \mathrm{kpc}$.

All our results provide evidence for an association of the two CHVCs with the Leading Arm of the Magellanic System at a distance of $d \approx 10 \ldots 60 \mathrm{kpc}$. This distance estimation allows us to constrain the physical parameters of the clouds and to learn more about the physical conditions in the environment of the Leading Arm.

To learn more about the nature of HVCs in general, it is necessary to make absorption measurements towards suitable background sources at known distance. This would provide a direct distance estimate as well as information about the metallicity of the clouds to confirm an association with the Magellanic Clouds. High-resolution observations of CHVCs in the vicinity of the Leading Arm and the Magellanic Stream are necessary to resolve other small-scale structures for a statistical analysis of core-halo structures in CHVCs. Furthermore, $\mathrm{H} \alpha$ measurements will be of great importance to investigate the interaction with an ambient medium in considerably more detail. Simulations are necessary for a better understanding of how such cold clumps are generated and to analyse the conditions under which interactions occur.

Acknowledgements. Many thanks to Philipp Richter for his helpful comments. Thanks to Benjamin Winkel for his helping hands. T.W. is supported by the DFG through grant KE 757/4-1.

\section{References}

Bland-Hawthorn, J., Veilleux, S., Cecil, G. N., et al. 1998, MNRAS, 299, 611 Blitz, L., Spergel, D. N., Teuben, P. J., Hartmann, D., \& Burton, W. B. 1999, ApJ, 514, 818

Braun, R., \& Burton, W. B. 1999, A\&A, 341, 437

Braun, R., \& Burton, W. B. 2000, A\&A, 354, 853 
Bregman, J. N. 1980, ApJ, 236, 577

Bregman, J. N. 2004, in High Velocity Clouds, ASSL, 312, 341

Brüns, C., \& Westmeier, T. 2004, A\&A, 426, L9

Brüns, C., Kerp, J., \& Staveley-Smith, L. 2000, in Mapping the Hidden Universe:

The Universe behind the Milky Way - The Universe in HI, ASP Conf. Ser., 218, 349

Brüns, C., Kerp, J., \& Pagels, A. 2001, A\&A, 370, L26

Brüns, C., Kerp, J., Staveley-Smith, L., et al. 2005, A\&A, 432, 45

Burton, W. B., Braun, R., \& Chengalur, J. N. 2001, A\&A, 375, 227

Cram, T. R., \& Giovanelli, R. 1976, A\&A, 48, 39

Danly, L., Albert, C. E., \& Kuntz, K. D. 1993, ApJ, 416, L29

de Heij, V., Braun, R., \& Burton, W. B. 2002, A\&A, 391, 159

Gardiner, L. T., \& Noguchi, M. 1996, MNRAS, 278, 191

Giovanelli, R., Verschuur, G. L., \& Cram, T. R. 1973, A\&AS, 12, 209

Greisen, E. W., \& Cram, T. R. 1976, ApJ, 203, L119

Högbom, J. A. 1974, A\&AS, 15, 417

Kalberla, P. M. W. 2003, ApJ, 588, 805

Kalberla, P. M. W., \& Haud, U. 2006, A\&A, 455, 481

Kalberla, P. M. W., Burton, W. B., Hartmann, D., et al. 2005, A\&A, 440, 775

Konz, C., Brüns, C., \& Birk, G. 2002, A\&A, 391, 713

Mathewson, D. S., Cleary, M. N., \& Murray, J. D. 1974, ApJ, 190, 291

Muller, C. A., Oort, J. H., \& Raimond, E. 1963, C. R. Acad. Sci. Paris, 257, 1661

Murphy, E. M., Lockman, F. J., \& Savage, B. D. 1995, ApJ, 447, 642
Oort, J. H. 1966, Bull. Astron. Inst. Netherlands, 18, 421

Putman, M. E., Gibson, B. K., Staveley-Smith, L., et al. 1998, Nature, 394, 752 Quilis, V., \& Moore, B. 2001, ApJ, 555, L95

Rasmussen, A., Kahn, S. M., \& Paerels, F. 2003, in The IGM/Galaxy Connection, The Distribution of Baryons at $z=0$, ed. J. L. Rosenberg, \& M. E. Putman, ASSL, 281, 109

Sembach, K. R., Wakker, B. P., Savage, B. D., et al. 2003, ApJS, 146, 165

Shapiro, P. R., \& Field, G. B. 1976, ApJ, 205, 762

Sternberg, A., McKee, C. F., \& Wolfire, M. G. 2002, ApJS, 143, 419

Thom, C., Putman, M. E., Gibson, B. K., et al. 2006, ApJ, 638, L97

van der Marel, R. P., Alves, D. R., Hardy, E., \& Suntzeff, N. 2002, AJ, 124, 2639

van Woerden, H., Schwarz, U. J., Peletier, R. F., Wakker, B. P., \& Kalberla, P. M. W. 1999, Nature, 400, 138

Vieser, W., \& Hensler, G. 2001, in Astronomische Gesellschaft Meeting Abstracts, ed. E. R. Schielicke, 106

Wakker, B. P. 1991, A\&A, 250, 499

Wakker, B. P., \& Boulanger, F. 1986, A\&A, 170, 84

Wakker, B. P., \& van Woerden, H. 1997, ARA\&A, 35, 217

Wakker, B. P., Oosterloo, T. A., \& Putman, M. E. 2002, AJ, 123, 1953

Westmeier, T., Brüns, C., \& Kerp, J. 2005, A\&A, 432, 937

Wolfire, M. G., McKee, C. F., Hollenbach, D., \& Tielens, A. G. G. M. 1995, ApJ, 453, 673

Yoshizawa, A. M., \& Noguchi, M. 2003, MNRAS, 339, 1135 